\title{
STRUCTURAL CHANGES EVIDENCED BY FTIR SPECTROSCOPY IN CELLULOSIC MATERIALS AFTER PRE-TREATMENT WITH IONIC LIQUID AND ENZYMATIC HYDROLYSIS
}

\author{
Iuliana Spiridon, Carmen-Alice Teacă, ${ }^{*}$ and Ruxanda Bodîrlău
}

\begin{abstract}
Attempts were made to enhance the hydrolysis of Asclepias syriaca (As) seed floss and poplar seed floss (PSF) by cellulase after pre-treatment with ionic liquids. Two ionic liquids, namely 1-butyl-3-methylimidazolium chloride $[\mathrm{BMIM}] \mathrm{Cl}$ and 1-ethyl-3-methylimidazolium tetrachloroaluminate $[\mathrm{EMIM}] \mathrm{Cl}-\mathrm{AlCl}_{3}$, were used. In comparison with conventional cellulose pretreatment processes, the ionic liquids were used under a milder condition corresponding to the optimum activity of cellulase. Hydrolysis kinetics of the IL-treated cellulose materials was significantly enhanced. The initial hydrolysis rates for IL-treated cellulose materials were greater than those of non-treated ones. The structural modifications of hydrolyzed cellulose materials were analyzed using FTIR spectroscopy.
\end{abstract}

Keywords: Cellulose material; Ionic liquid; Enzymatic hydrolysis; FTIR spectroscopy

Contact information: Romanian Academy, "Petru Poni" Institute of Macromolecular Chemistry, 41 A Gr. Ghica Voda Alley, Iasi, RO-700487, Romania

"Corresponding author: cateaca@icmpp.ro, cateaca14@yahoo.com

\section{INTRODUCTION}

Cellulose is a major fraction of plant biomass. It is the most abundant renewable resource in the world, and it can be useful in the conventional petrochemical refineries in an economy based on renewable resources (Klemm 2005; Lynd et al. 1999). In its natural state, cellulose is highly crystalline in structure with individual cellulose polymer chains held together by strong hydrogen bonding and van der Waals forces. The individual cellulose chains are a linear condensation polymer molecules made up of anhydroglucose units joined together by $\beta-1,4$-glycosidic bonds (Klemm et al. 1998) with degrees of polymerization ranging from 1000 to 15,000 units. In general, neither the water molecules nor the catalysts for hydrolysis (like cellulase enzymes) are able to easily penetrate the crystalline matrix (Zhang and Lynd 2004; Lynd et al. 2002). Enzymatic saccharification processes require the dissolution of cellulose in a solvent to facilitate the access of cellulase to cellulosic substrates. Cellulose consists of polydispersed linear polymer chains that form hydrogen-bonded supramolecular structures; because of this, cellulose is insoluble in water and most common organic liquids.

Ionic liquids (IL's), a new class of cellulose-dissolving solvents (Swatloski et al. 2002), and new reaction media for biocatalysis (Van Rantwijk and Sheldon 2007; Lau et al. 2004), are potential solvents for the enzymatic saccharification of cellulose. However, the significant decrease in cellulase activity in the presence of cellulose-dissolving IL's 
(Turner et al. 2003) requires that a cumbersome recovery process is necessary to retrieve the regenerated cellulose produced by the pretreatment of cellulose with IL's prior to enzymatic saccharification (Dadi et al. 2006). To simplify the entire process, it is necessary to develop IL's that are compatible with both cellulose solubility and cellulase activity.

The application of ionic liquids (IL's) as solvents in carbohydrates chemistry (Murugesan and Linhardt 2005; Zhu et al. 2006) or reaction medium for the homogeneous cellulose modification (Liebert and Heinze 2008) has recently been reviewed. Some ionic liquids, especially those containing the $\mathrm{Cl}^{-}$anion, are capable of dissolving cellulose (Cao et al. 2009). Ionic liquids have the ability to dissolve large amounts of cellulose under relatively mild conditions, and the feasibility of recovering nearly $100 \%$ of the used IL to its initial purity makes them attractive (Dadi et al. 2006; Heinze et al. 2005; Dadi et al. 2007).

Cellulose can be dissolved in methylimidazolium salts, especially 1-butyl- 3methylimidazolium chloride ([BMIM]Cl) (Swatloski et al.2002; Swatloski et al. 2003; Mazza et al. 2009) and 1-allyl-3-methylimidazolium chloride ([AMIM]Cl) (Wu et al. 2004; Zhang et al. 2005) ammonium salts such as benzyldimethyl(tetradecyl)ammonium chloride ([BDTA]Cl), and pyridinium salts such as 1-butyl-3-methylpyridinium chloride ([BMPy]Cl) (Heinze et al. 2005; El Seoud et al. 2007). In all these cases, the dissolution of the cellulose materials is also due to the action of the chloride anions, which interact with the hydroxyl groups of cellulose by disrupting the hydrogen bonds in the biopolymer (Swatlovski et al. 2002; Moulthrop et al. 2005). These interactions were evidenced by NMR method in solutions of cellulose in [BMIM]Cl at $5 \%$ at $90^{\circ} \mathrm{C}$ (Remsing et al. 2006; Moulthrop et al. 2005).

Acid and basic ionic liquids represent new classes of IL's (Olivier-Bourbigou et al. 2010). The acid or basic function can be attached either on the anion or on the cation (MacFarlane et al. 2006). IL's containing polynuclear metallic anions such as chloroaluminates have been known for a long time for their potential Lewis acidity and superacidity in the presence of protons. Many ionic liquids based on metal ions have been developed (Lin and Vasam 2005). Work has been first focused on chloroaluminates associated with imidazolium or pyridinium cations. A variety of different anions are formed in solution $\left(\left[\mathrm{AlCl}_{4}\right]^{-},\left[\mathrm{Al}_{2} \mathrm{Cl}_{7}\right]^{-},\left[\mathrm{Al}_{3} \mathrm{Cl}_{10}\right]^{-}\right)$, the ratio of which vary with changing aluminium chloride composition. These ideas have more recently been extended to other chlorometalate salts.

The use of ionic liquids in biocatalytic transformations has solved some of the problems encountered in their applications in aqueous and organic solvents. Ionic liquids are a particularly attractive alternative medium for the enzymatic reactions of carbohydrates, because their solubility is enhanced in ionic liquids.

Formulators of cellulose chemicals and soft fibrous structures are always looking for additional types of fibers in order to improve performance or reduce cost. Soft fibrous structures have conventionally been made with wood pulp fibers. More recently, synthetic fibers have been used. Seed hairs may protect a seed and/or aid in the transport of a seed present on a plant. Methods of separating and cleaning cotton staple fibers and/or cotton linters fibers, for example, are well known and effective. However, they are unsuitable for use with other types of seeds. 
Individualized seed hairs may be converted into chemical derivatives including but not limited to cellulose derivatives. Individualized seed hairs may also be used in their physical form, usually fibrous, and herein referred to "seed hair fibers", as a component of fibrous structures. Many plants have seed hairs. Seed hairs may have a wide range of morphology and chemical properties. For example, the seed hairs may be in the form of fibers, namely seed hair fibers. Such seed hair fibers may have a high length to diameter ratio.

Milkweed (Asclepias syriaca) is an industrial crop. The seed pods are harvested for their floss, which has been found to provide high thermal insulation, being produced commercially as hypoallergenic fiber fillers in comforters and pillows. Milkweed seeds end up as by-products of floss production and have limited applications as plants for landscaping and erosion control. Seeds contain $21 \%$ oil and $32 \%$ crude protein (dry basis) (Evangelista 2007). Milkweed seed protein has functional properties that may find use as a thickener, protein extender in adhesives, or emulsifier in paints (Evangelista et al. 2009). Milkweeds produce some kinds of fibers in their stems: the long, quite strong but brittle bast fiber and the seed hairs. These fibers can be used as a substitute for wood in pressed panels. It was also reported that it is possible to use Asclepias syriaca fibers for paper manufacture (Spiridon 2007; Popa et al. 1996).

In the present paper, structural changes of cellulose materials (Asclepias syriaca fibers, poplar seed floss), previously treated with two ionic liquids, including 1- $n$-butyl-3methylimidazolium chloride ([BMIM]Cl) and 1-ethyl-3-methylimidazolium tetrachloroaluminate $[\mathrm{EMIM}] \mathrm{Cl}-\mathrm{AlCl}_{3}$, were analyzed after enzymatic hydrolysis with cellulase. The hydrolysis rate was estimated by determination of the soluble sugars released during saccharification. The structural modifications of cellulose materials were evidenced by Fourier transform infrared spectroscopy (FTIR).

\section{EXPERIMENTAL}

\section{Materials}

Acetic acid, sodium acetate dihydrate, 3, 5-dinitrosalicylic acid (DNS), sodium hydroxide, sodium potassium tartrate (Rochelle salt), phenol, sodium metabisulfite, and ethanol were obtained from Sigma-Aldrich, Ltd. (Germany). Ionic liquids [BMIM]Cl IL1 and [EMIM]Cl-AlCl - IL2 (Fig. 1), > 95\% purity degree, were obtained from Fluka and have been used without further purification. Cellulase from Aspergillus niger strain was supplied by BioChemika, Fluka. Cellulose materials, the Asclepias syriaca seed floss (As) and poplar seed floss (PSF), were used without any preliminary treatment (Fig. 2).

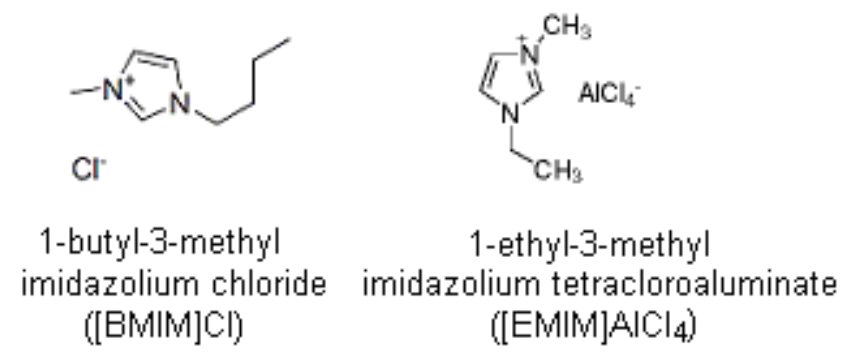

Fig.1. Structure of ionic liquids (IL's) used in the experiments 

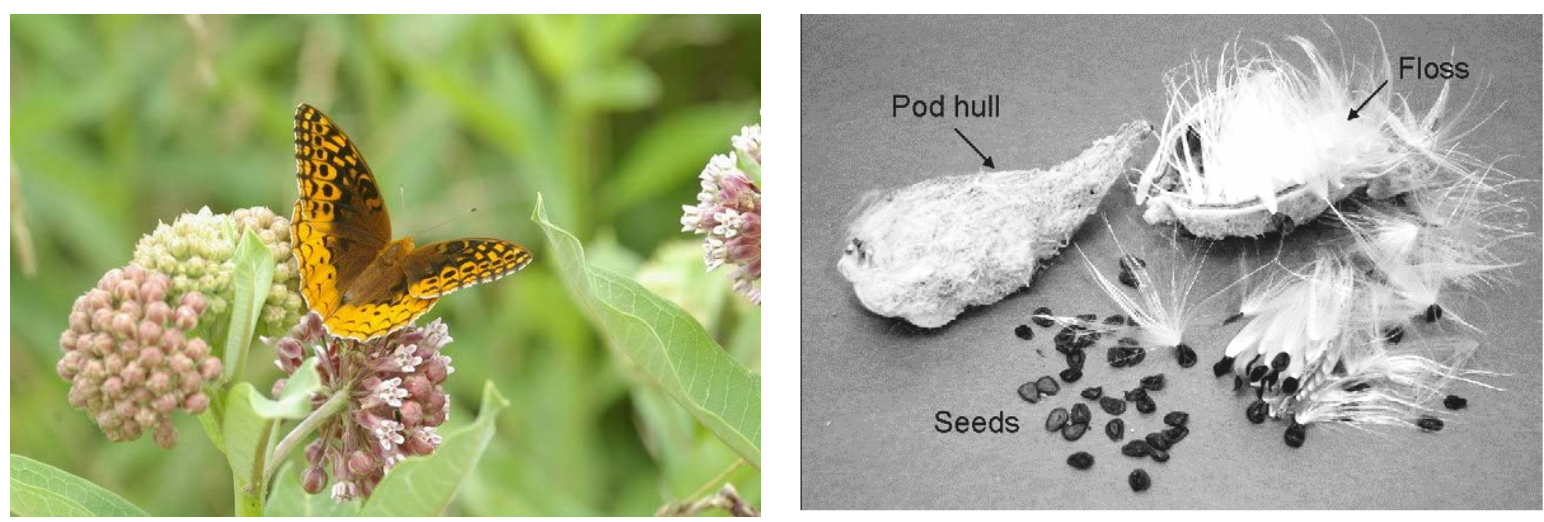

Fig. 2. Photographs of milkweed plant flowers and dried milkweed pod showing pod hull, floss, and seeds

\section{Methods}

Cellulose materials treatment with ionic liquids

The cellulose materials Asclepias syriaca seed floss (As) and poplar seed floss (PSF) were kept in a vaccum oven at $50{ }^{\circ} \mathrm{C}$ for $24 \mathrm{~h}$ prior to use, then were mixed with vacuum-dried ionic liquids IL1, respectively IL2 [material:solvent $=1: 10(\mathrm{w} / \mathrm{w})]$ into dried glass conical vessels equipped with mechanical stirrer, under an inert atmosphere of nitrogen. We should mention that [EMIM]Cl- $\mathrm{AlCl}_{3}$ (IL2) is very hygroscopic, and this may pose severe practical limitations in its handling and storage. Water impurities are known to react with the anions, releasing super-acidic protons. These cause unwanted side reactions in many applications and possess considerable potential for corrosion. Therefore, in order to minimize this detrimental action, IL2 was previously stored in a vacuum desiccator containing phosphorus pentoxide. The conical vessels containing a mixture of cellulose material and IL were then loosely capped, and maintained at $100^{\circ} \mathrm{C}$ for $10 \mathrm{~h}$, using an oil bath. The treated cellulose materials were washed with sufficient deionized water and dried at $70^{\circ} \mathrm{C}$ for $48 \mathrm{~h}$ before being subjected to enzymatic hydrolysis.

\section{Enzymatic hydrolysis of cellulose materials}

The cellulose substrates, As and PSF, were hydrolyzed without or previously treated with ionic liquid in vials using a waterbath WNB 7-45. The total vial volume was $5 \mathrm{~mL}$, containing cellulose material concentration of $2 \mathrm{mg} / \mathrm{mL}$. A cellulase addition of 30 $\mathrm{FPU} / \mathrm{g}$ was used in the hydrolysis reaction. The mixture was buffered with $50 \mathrm{mM}$ acetic acid-sodium acetate solution, $\mathrm{pH} 4.8$. The hydrolysis reaction was carried out at $40^{\circ} \mathrm{C}$ for $100 \mathrm{~h}$. All cellulose material samples were hydrolyzed using the same cellulase stock solution. The release of soluble reducing sugars was periodically measured by the DNS assay.

\section{Analysis}

Chemical analysis of As and PSF was performed according to the TAPPI methods. The milkweed floss is composed of $54.9 \%$ cellulose, $8.0 \%$ hemicelluloses, 
$19.3 \%$ lignin, and $0.9 \%$ ash. The poplar seed floss is composed of $62.07 \%$ cellulose, $17.04 \%$ lignin, and $2.5 \%$ ash.

Reducing sugar was measured by the DNS assay (Miller 1959). Glucose was used as a standard for the reducing sugar measurement. Triplicate determinations were made for each hydrolysate sample. Cellulase activity was determined by the standard filter paper assay and expressed as filter paper units per gram (FPU) (Ghose 1987). One FPU is defined as the enzyme that releases $1 \mu \mathrm{mol}$ of glucose equivalents per minute from Whatman No.1 filter paper.

The color intensity of the hydrolysate samples was measured in a Cole-Palmer spectrophotometer at $540 \mathrm{~nm}$. The hydrolysis rate of reducing sugars from treated cellulose materials was calculated with Eq. (1) as follows:

Hydrolysis rate $(\%)=$

(reducing sugars weight/dry cellulose material weight $) \times 100$

Fourier transformed infrared spectroscopy (FTIR) was performed by using a Bruker Vertex 70 spectrophotometer. The spectra $\left(4000-400 \mathrm{~cm}^{-1}\right)$ were recorded with a resolution of $4 \mathrm{~cm}^{-1}$ and 64 scans per sample. About $2.0 \mathrm{mg}$ samples were prepared by mixing with $120 \mathrm{mg}$ of spectroscopic grade $\mathrm{KBr}$ then pressed to produce $13 \mathrm{~mm}$ diameter pellets.

\section{RESULTS AND DISCUSSION}

\section{Cellulase-Catalyzed Hydrolysis of Cellulose Materials}

It has been shown that the structure of cellulose material regenerated from used ionic solutions by addition of water is less crystalline when compared to the original untreated cellulose (Biganska and Navard 2009).

The solubility of cellulose, lignin, and carbohydrates in the ionic liquids has been studied (Egorov et al. 2007). Overall, the ability of IL's to dissolve cellulose material depends on its nature (degree of polymerization of cellulose component, crystallinity), the operating conditions (reaction time, temperature, initial concentration of cellulose material in the IL), and the presence of impurities.

The influence of IL's used for treatment of cellulose materials (As, PSF) on their further enzymatic hydrolysis with cellulase was evaluated. The cellulose-enriched materials solubilization in ionic liquid modified the ordered structure of substrate with a positive impact on the rates of enzymatic hydrolysis. [BMIM]Cl seemed to be a noticeably better solvent, while [EMIM]Cl-AlCl 3 caused a more intense degradation process of the cellulose materials through increasing of reaction medium acidity. It was found that the enzymatic hydrolysis rate was improved significantly by pre-treatment with ionic liquid (Fig. 3-a, b).

It seems that the cellulose samples exhibiting lower crystallinity and higher cellulase adsorption were hydrolyzed by cellulase much faster after treatment with IL's, compared with samples untreated with IL's. The hydrolysis rate of As pretreated with IL1 
was only $8.8 \%$ after 50 hours of enzymatic hydrolysis, greater than that obtained for AsIL2. However, the hydrolysis rate had a value of $17.35 \%$ for PSF when it was pretreated with [BMIM]Cl and $12.55 \%$ for PSF-IL2, respectively.

Cellulose material represented by As presents a more compact structure comparatively with PSF, and this fact can diminish the rate of the cellulose hydrolysis reaction. It is well known that use of IL's for pre-treatment of cellulose materials may cause some degradation of components. Hemicelluloses present in As contain carboxylic acid moieties that may induce such degradation reactions. Nevertheless, IL's themselves exhibit some catalytic action under acidic conditions. For chloride-based imidazolium IL's, the proton present on the 2-position of the imidazole ring acts as a hydrogen-bond donor, and the associated chloride acts as a hydrogen-bond acceptor. This can effectively activate the hydroxyl groups of carbohydrates dissolved in IL's (Moulthrop et al. 2005; Mazza et al. 2009). The increase of reaction medium acidity during cellulose materials pre-treatment with $[\mathrm{EMIM}] \mathrm{Cl}-\mathrm{AlCl}_{3}$ can depolymerize cellulose and hemicelluloses by hydrolysis to smaller fractions.
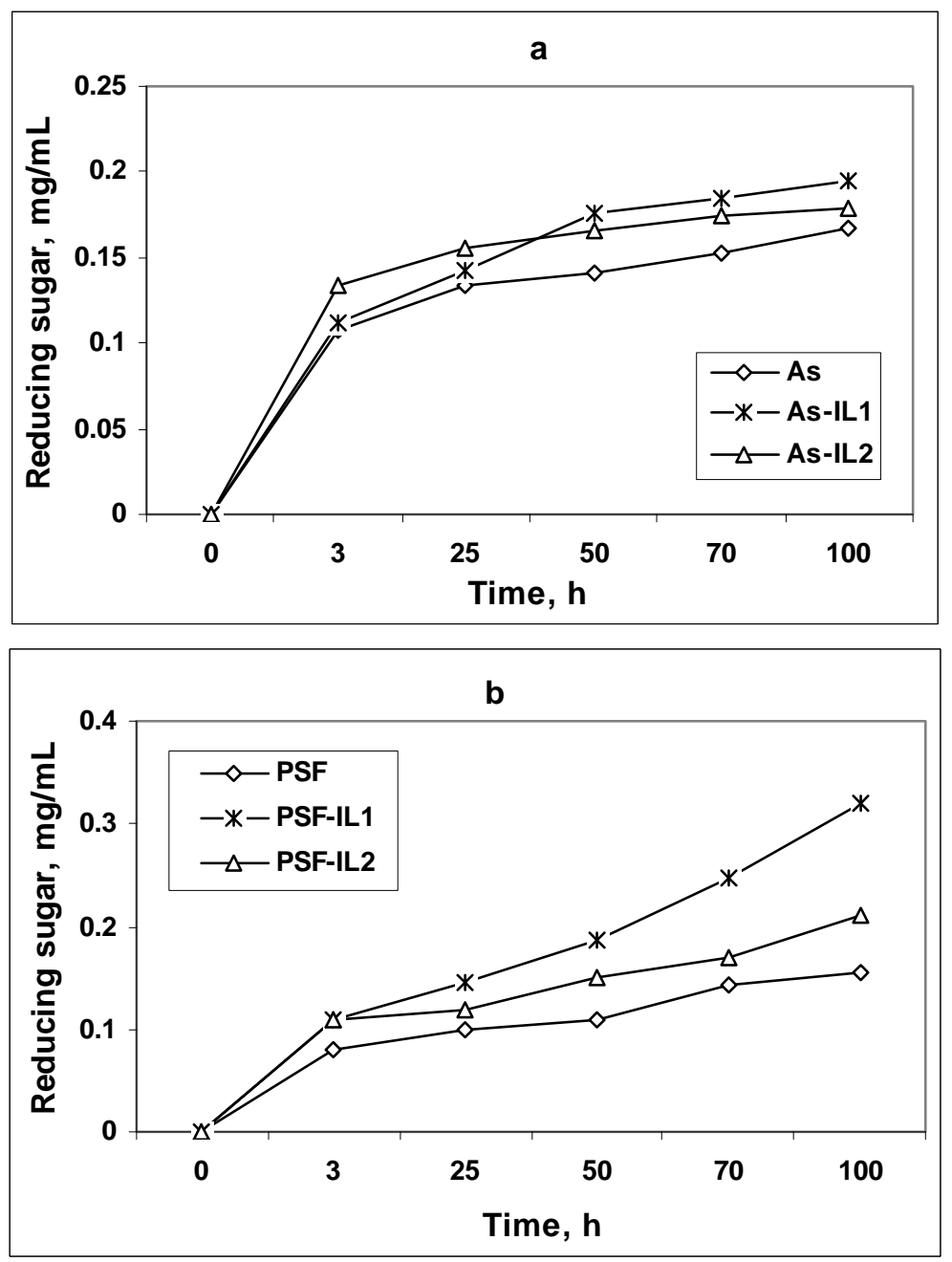

Fig.3. Reducing sugar released during enzymatic hydrolysis of untreated and pretreated cellulose materials: a) As, and b) PSF 
The hydrolysis rate of untreated As increased up to $8.35 \%$ after $100 \mathrm{~h}$. This behavior may be related to the incomplete accessibility of the cellulose fraction to cellulase, and thus limiting enzymatic hydrolysis. However, the hydrolysis rate of As and PSF treated with [BMIM]Cl was significantly improved after $40 \mathrm{~h}$. After $100 \mathrm{~h}$ of enzymatic reaction, the hydrolysis rate of substrates after treatment with [BMIM]Cl was greater than that obtained in the case of $[\mathrm{EMIM}] \mathrm{Cl}-\mathrm{AlCl}_{3}$ treatment. It seems that treatment of As and PSF with IL's increased the accessible fraction of cellulose. Differences appeared by using these two IL's could be due to the significant degradation process of both cellulose materials by treatment with [EMIM]Cl-AlCl 3 . The loss of intraand inter-molecular hydrogen bonding resulting in amorphous cellulose provided an enhanced surface area, leading to better enzyme accessibility and increased binding sites in recovered cellulose materials. The difference in the hydrolysis rates and amounts of total reducing sugars released from two pre-treatments can be attributed to the differences in the ensuing cellulose structures of amorphous cellulose from [BMIM]Cl pre-treatment and largely crystalline cellulose from [EMIM]Cl-AlCl ${ }_{3}$ pre-treatment ( $\mathrm{Li}$ et al. 2009; Liu and Chen, 2006; Zhao et al. 2009).

\section{FTIR Spectroscopy Analysis of Cellulose Materials}

Fourier transformed IR spectra in the $800-1800 \mathrm{~cm}^{-1}$ region were employed to characterize the structure of cellulose materials under study. Figures 4 and 5 present the FTIR spectra of cellulose materials before and after pre-treatment with ionic liquid. The absorption band at $1430 \mathrm{~cm}^{-1}$ is assigned to the $\mathrm{CH}_{2}$ scissoring motion in cellulose. The absorption band at $897 \mathrm{~cm}^{-1}$ is assigned as $\mathrm{C}-\mathrm{O}-\mathrm{C}$ stretching at the $\beta$ - $(1 \rightarrow 4)$-glycosidic linkage in cellulose (Nelson and O'Connor 1964).
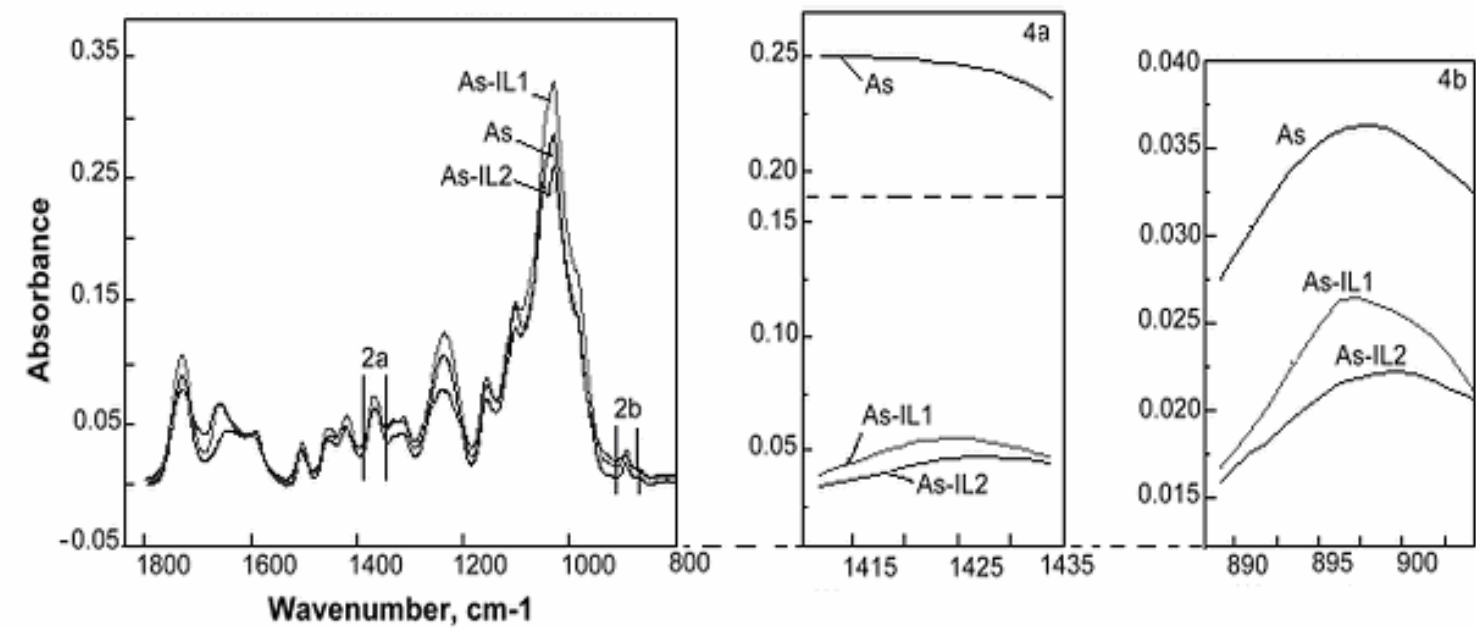

Fig. 4. FTIR spectra for As before and after pre-treatment with ionic liquids: a) $1400-1435 \mathrm{~cm}^{-1}$ region; b) $800-1000 \mathrm{~cm}^{-1}$ region 


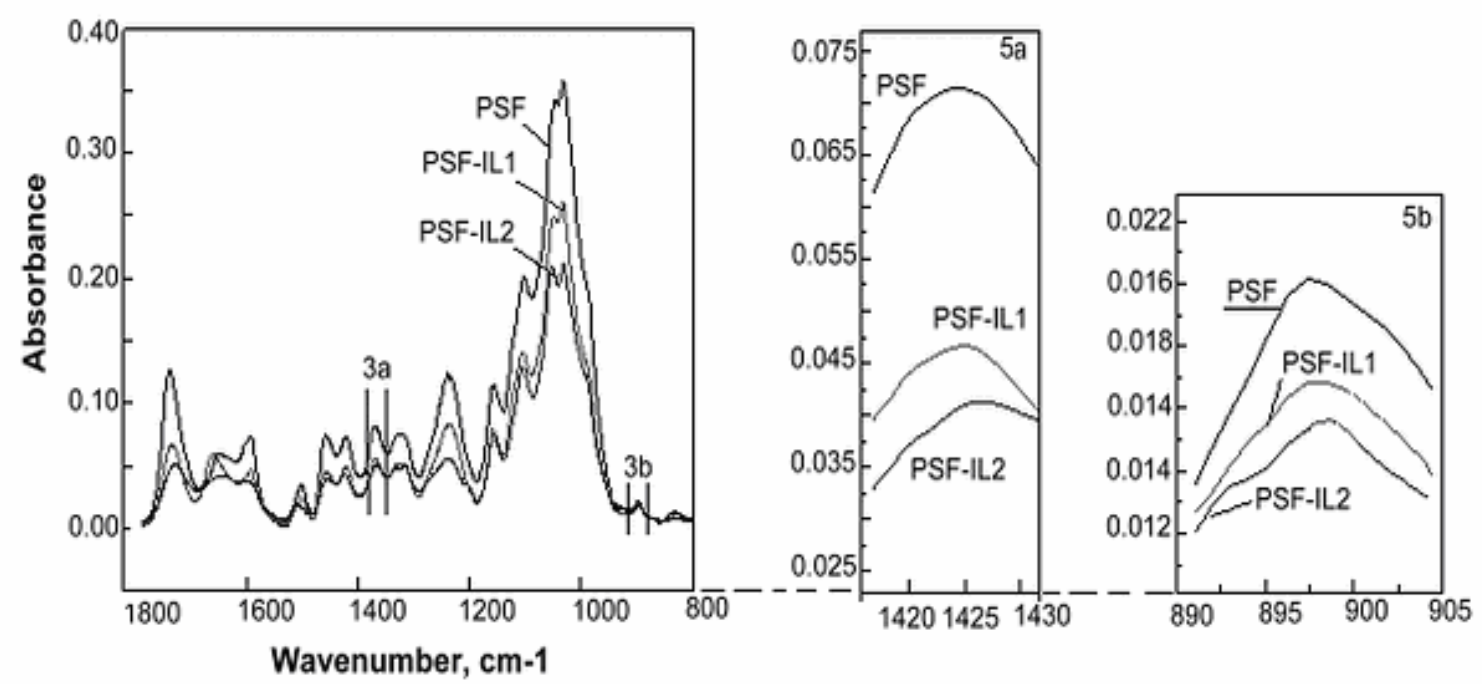

Fig. 5. FTIR spectra for PSF before and after treatment with ionic liquids: a) $1415-1430 \mathrm{~cm}^{-1}$ region; b) 890-905 $\mathrm{cm}^{-1}$ region

As shown in Fig. 4, the absorption band at $1430 \mathrm{~cm}^{-1}$ was strong for untreated cellulose materials, but weak for the treated ones. The spectra shown in Figs. 4 and 5 indicate that the untreated cellulose materials As and PSF possess cellulose I crystal type. This is in accord with the well-known fact that almost all native celluloses in the higher plants have the crystal structure of cellulose I. The cellulose I structure was transformed into amorphous or cellulose II structure after treatment with ionic liquid and enzymatic hydrolysis (Figs. 6 and 7).
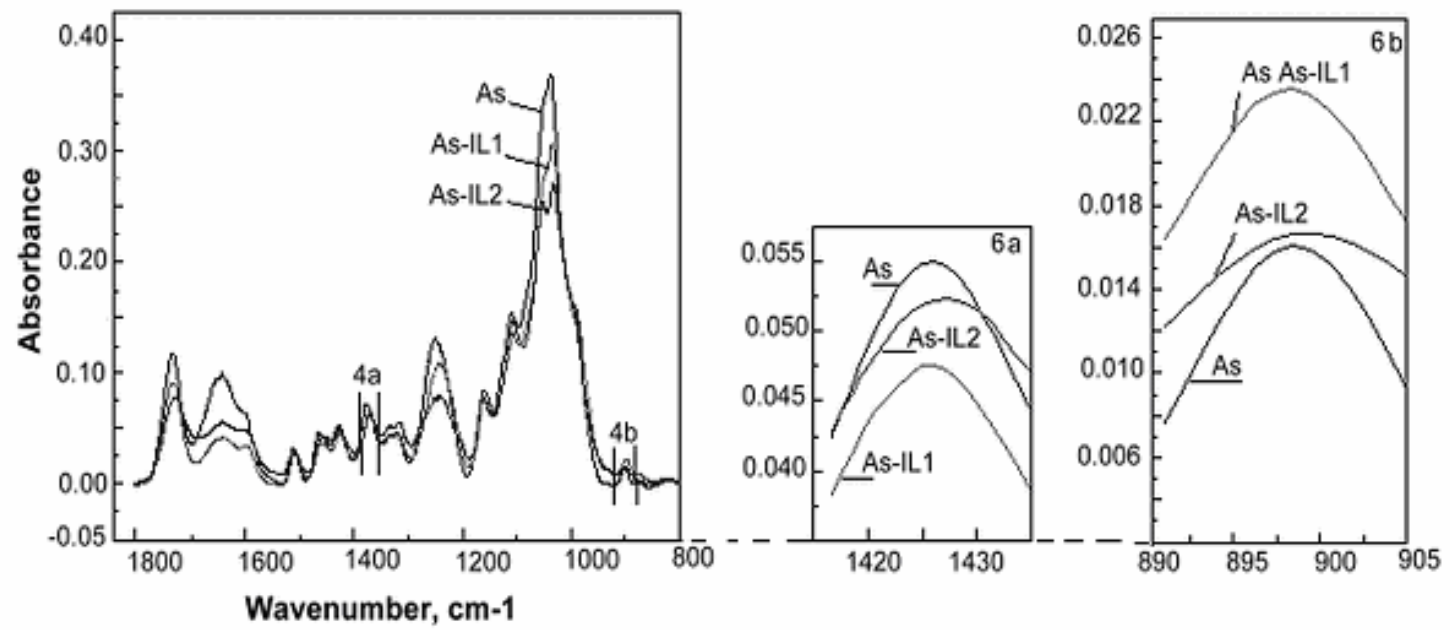

Fig. 6. FTIR spectra for As after treatment with ionic liquids and enzymatic hydrolysis: a) 1415$1435 \mathrm{~cm}^{-1}$ region; b) 890-905 $\mathrm{cm}^{-1}$ region 


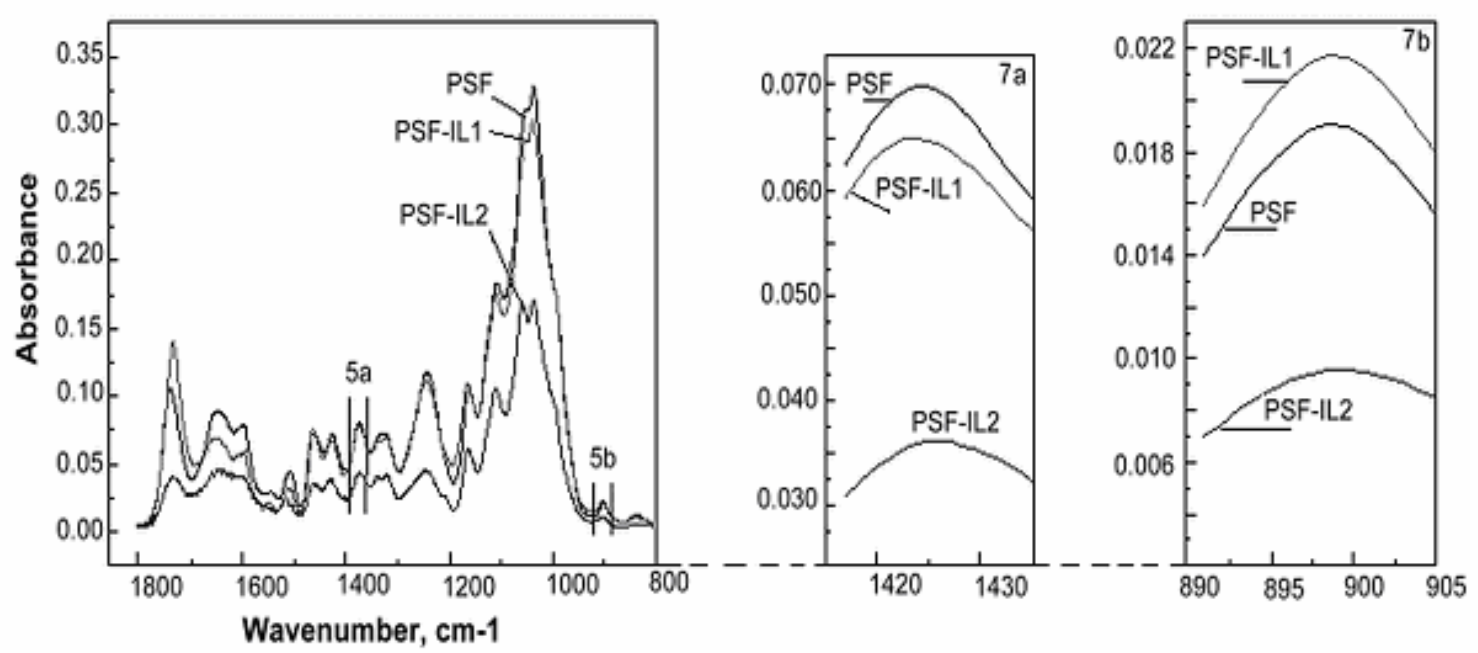

Fig.7. FTIR spectra for PSF after treatment with ionic liquids and enzymatic hydrolysis: a) 1415$1435 \mathrm{~cm}^{-1}$ region; b) 890-905 $\mathrm{cm}^{-1}$ region

The absorbance values at $1430 \mathrm{~cm}^{-1}$ and $897 \mathrm{~cm}^{-1}$ are quite sensitive to the crystal structure of cellulose in cellulosic materials. Thus, the absorbance ratio $\mathrm{A}_{1430} / \mathrm{A}_{897}$, which is known as crystallinity index (O'Connor et al. 1958) or lateral order index (LOIHurtubise and Kräsig 1960), has been used as evidence of the presence of cellulose I fraction in the cellulose material structure (Oh et al. 2005). The FTIR spectra show characteristic cellulose peaks around $1000-1200 \mathrm{~cm}^{-1}$ (Zhbankov et al. 2000; Langkilde and Svantesson 1995). The band near $1160 \mathrm{~cm}^{-1}$ is representative of the anti-symmetric bridge stretching of C-O-C groups, and the band near $1318 \mathrm{~cm}^{-1}$ can be ascribed to $\mathrm{CH}_{2}$ wagging vibrations in cellulose.

The results of FTIR investigation showed that the peaks of cellulose materials were lower after enzymatic hydrolysis and especially after treatment with ionic liquids, indicating that some of cellulose was degraded. The band at $1635-1640 \mathrm{~cm}^{-1}$, which has been attributed to the absorbed water bending vibrations, significantly decreased after enzymatic hydrolysis after pretreatment with ionic liquids. Another two infrared ratios were calculated: (1) $\mathrm{A}_{1372} / \mathrm{A}_{2900}$, which is known as the total crystallinity index (TCI) (Nelson and O'Connor 1964), and (2) $\mathrm{A}_{3308} / \mathrm{A}_{1330}$, which is known as hydrogen-bond intensity (HBI) (Oh et al. 2005; Šroký et al. 2010). These parameters, shown in Tables 1 and 2, are closely related to the crystal system and degree of the intermolecular regularity.

A higher index value (TCI, LOI) indicates that the material has a high crystallinity and an ordered structure. This indicates that a part of the crystalline structure of cellulose was transformed into amorphous form through treatment with IL's (Li et al. 2010). As a consequence, the fragmental and porous treated cellulose materials with highly amorphous structure could provide more surfaces for enzymes to attack on. This fact is confirmed by enzymatic hydrolysis rate of cellulose material samples treated with ionic liquid. 
LOI and HBI values decrease for both cellulose materials treated with IL's by comparison with the untreated ones (Table 1). Probably, during the pre-treatment with IL's, the cellulose materials lost their crystalline structure and, instead, restructured themselves into mostly amorphous forms. This decrease in cellulose crystallinity index (LOI) implies that the treated cellulose material has an increase in cellulose surface accessibility and would theoretically enable more efficient cellulase hydrolysis.

HBI values decrease for all samples (As, PSF) treated with both IL's, while LOI parameter exhibits a different behavior as it decreases for As-IL1 and PSF-IL1 and increases for AS-IL2.and PSF-IL2 after enzymatic hydrolysis (see Tables 1 and 2). This fact may be related to the initial characteristics of cellulose materials subjected to IL's pre-treatment (As is a more dense cellulose material than PSF), as well as to the specific action of IL2 ([EMIM]Cl- $\left.\mathrm{AlCl}_{3}\right)$ that may significantly aid cellulose degradation during pre-treatment.

Table 1. Crystallinity Indices for Cellulose Materials before and after Treatment with Ionic Liquids

\begin{tabular}{|c|c|c|c|}
\hline $\begin{array}{c}\text { Cellulose } \\
\text { material }\end{array}$ & $\begin{array}{c}\text { LOI } \\
\left(1437 \mathrm{~cm}^{-1} / 899 \mathrm{~cm}^{-1}\right)\end{array}$ & $\begin{array}{c}\mathrm{TCl} \\
\left(1378 \mathrm{~cm}^{-1} / 2900 \mathrm{~cm}^{-1}\right)\end{array}$ & $\begin{array}{c}\mathrm{HBI} \\
\left(3308 \mathrm{~cm}^{-1} / 1330 \mathrm{~cm}^{-1}\right)\end{array}$ \\
\hline As & 2.24 & 0.77 & 5.29 \\
\hline As-IL1 & 2.15 & 0.75 & 4.80 \\
\hline As-IL2 & 2.06 & 0.82 & 3.90 \\
\hline PSF & 2.58 & 0.49 & 4.42 \\
\hline PSF-IL1 & 2.41 & 0.73 & 4.38 \\
\hline PSF-IL2 & 2.35 & 0.79 & 4.37 \\
\hline
\end{tabular}

Table 2. Crystallinity Indices for Cellulose Materials after Treatment with Ionic Liquids and Enzymatic Hydrolysis

\begin{tabular}{|c|c|c|c|}
\hline $\begin{array}{c}\text { Cellulose } \\
\text { material }\end{array}$ & $\begin{array}{c}\text { LOI } \\
\left(1437 \mathrm{~cm}^{-1} / 899 \mathrm{~cm}^{-1}\right)\end{array}$ & $\begin{array}{c}\mathrm{TCl} \\
\left(1378 \mathrm{~cm}^{-1} / 2900 \mathrm{~cm}^{-1}\right)\end{array}$ & $\begin{array}{c}\mathrm{HBI} \\
\left(3308 \mathrm{~cm}^{-1} / 1330 \mathrm{~cm}^{-1}\right)\end{array}$ \\
\hline As & 1.97 & 0.71 & 5.80 \\
\hline As-IL1 & 1.98 & 0.93 & 4.77 \\
\hline As-IL2 & 2.95 & 0.79 & 3.52 \\
\hline PSF & 2.74 & 0.54 & 4.42 \\
\hline PSF-IL1 & 2.02 & 0.36 & 4.01 \\
\hline PSF-IL2 & 2.65 & 0.71 & 4.23 \\
\hline
\end{tabular}

The values of parameters were influenced by treatment with ionic liquid and enzymatic hydrolysis of cellulose materials under study. Thus, the ordered well crystalline phase and the degree of intermolecular regularity of carbohydrates from substrate considered in this study were affected by treatment with IL's through changing the fiber structure.

\section{CONCLUSIONS}

1. The present paper is dedicated to study the hydrolysis using as substrate the Asclepias syriaca seed floss (As), and poplar seed floss (PSF), which are cellulose-enriched 
materials. This is in agreement with the current tendency to prioritize developing processes under environmentally friendly conditions.

2. Cellulose materials originating from Asclepias syriaca seed floss and poplar seed floss treated with ionic liquid and further hydrolyzed with cellulase were hydrolyzed more easily than those hydrolyzed without pre-treatment. Treatment with IL's modified the ordered structure of substrates with a positive impact on the rates of enzymatic hydrolysis by increasing the accessible fraction of cellulose. Differences appeared by using these two IL's could be due to the significant degradation process of both cellulose materials by pre-treatment with [EMIM]Cl-AlCl ${ }_{3}$ through increasing of reaction medium acidity which can depolymerize cellulose and hemicelluloses by hydrolysis to smaller fractions.

3. The loss of intra- and inter-molecular hydrogen bonding, resulting in amorphous cellulose, provided an enhanced surface area, leading to better enzyme accessibility and increased binding sites in recovered cellulose materials. The difference in the hydrolysis rates and amounts of total reducing sugars released from two pretreatments can be attributed to the differences in the ensuing cellulose structures of amorphous cellulose from [BMIM]Cl pre-treatment and largely crystalline cellulose from [EMIM]Cl-AlCl ${ }_{3}$ pre-treatment.

4. FTIR investigation evidenced that the peaks of cellulose materials were lower after enzymatic hydrolysis and especially after treatment with ionic liquids, indicating that some of cellulose was degraded. Higher index values obtained from calculated infrared ratios (total crystallinity index TCI, lateral order index LOI) indicate that the cellulose materials have a high crystallinity and an ordered structure. A part of the crystalline structure of cellulose was transformed into amorphous form through pretreatment of cellulose materials with IL's.

5. Decrease in cellulose crystallinity index (LOI) indicates that treated cellulose material has an increase in cellulose surface accessibility and would theoretically enable more efficient cellulase hydrolysis. Differences should be expected when using different IL's, mainly in the case of those which may act detrimentally to the cellulose fraction from cellulosic materials.

\section{REFERENCES CITED}

Biganska, O., and Navard, P. (2009). "Morphology of cellulose objects regenerated from cellulose-N-methylmorpholine N-oxide-water solutions," Cellulose 16, 179-188.

Cao, Y., Wu, J., Zhang, J., Li, H., Zhang, Y., and He, J. (2009). "Room temperature ionic liquids (RTILs): A new and versatile platform for cellulose processing and derivatization," Chem. Eng. J. 147, 13-21.

Dadi, A. P., Varanasi, S., and Schall, C. A. (2006). "Enhancement of cellulose saccharification kinetics using an ionic liquid pretreatment step," Biotechnol. Bioeng. 95, 904-910.

Dadi, A. P., Schall, C. A., and Varanasi, S. (2007). "Mitigation of cellulose recalcitrance to enzymatic hydrolysis by ionic liquid pretreatment," Appl. Biochem. Biotechnol. $137-140,407-421$. 
Egorov, V. M., Smirnova, S. V., Formanovsky, A. A., Pletnev, I. V., and Zolotov, Y. A. (2007). "Dissolution of cellulose in ionic liquids as a way to obtain test materials for metal-ion detection," Anal. Bioanal. Chem. 387, 2263-2269.

El Seoud, O. A., Koschella, A., Fidale, L. C., Dorn, S., and Heinze, T. (2007).

"Applications of ionic liquids in carbohydrate chemistry: A window of opportunities," Biomacromolecules 8, 2629-2647.

Evangelista, R. L. (2007). "Milkweed seed wing removal to improve oil extraction," Ind. Crops Prod. 25, 210-217.

Evangelista, M. P. H., Evangelista, R. L., and Wu, Y. V. (2009). "Characterization of milkweed (Asclepias spp.) seed proteins," Ind. Crops Prod. 29, 275-280.

Ghose, T. K. (1987). "Measurement of cellulase activities," Pure Appl. Chem. 59, 257268.

Heinze, T., Schwikal, K., and Barthel, S. (2005). "Ionic liquids as reaction medium in cellulose functionalization," Macromol. Biosci. 5, 520-525.

Hurtubise, F. G., and Kräsig, H. (1960). "Classification of fine structural characteristics in cellulose by infrared spectroscopy. Use of potassium bromide pellet technique," Anal. Chem. 32, 177-181.

Klemm, D., Philip, B., Heinze, T., Heinze, U., and Wagenknecht, W. (1998). Comprehensive Cellulose Chemistry, Volume 1. Fundamentals and Analytical Methods, Wiley-VCH, Weinheim.

Klemm, D., Heublein, B., Fink, H.-P., and Bohn, A. (2005). "Cellulose: Fascinating biopolymer and sustainable raw material," Angew. Chem. Int. 44, 2-37.

Langkilde, F. W., and Svantesson, A. (1995). "Identification of celluloses with Fouriertransform (FT) mid-infrared, FT-Raman and near-infrared spectrometry," J. Pharm. Biomed. Anal. 13, 409-414.

Lau, R. M., Sorgedrager, M. J., Carrea, G., van Rahtwijk, F., Secundo, F., and Sheldon R. A. (2004). "Dissolution of Candida antarctica lipase B in ionic liquids: Effects on structure and activity," Green Chem. 6, 483-487.

Liebert, T., and Heinze, T. (2008). "Interaction of ionic liquids with polysaccharides. 5. Solvents and reaction media for the modification of cellulose," BioResources (http://ncsu.edu/bioresources), 3(2), 576-601.

Li, B., Filpponen, I., and Argyropoulos, D. S. (2010). "Acidolysis of wood in ionic liquids," Ind. Eng. Chem. Res. 49, 3126-3136.

Li, Q., He, Y.-C., Xian, M., Jun, G., Xu, X., Yang, J.-M., and Li, L.-Z., (2009). "Improving enzymatic hydrolysis of wheat straw using ionic liquid 1-ethyl-3-methyl imidazolium diethyl phosphate pretreatment," Biores. Technol. 100, 3570-3575.

Lin, I. J. B., and Vasam, C. S. (2005). "Metal-containing ionic liquids and ionic liquid crystals based on imidazolium moiety," J. Organomet. Chem. 690, 3498-3512.

Liu, L., and Chen, H. (2006). "Enzymatic hydrolysis of cellulose materials treated with ionic liquid [BMIM] Cl," Chin. Sci. Bull. 51, 2432-2436.

Lynd, L. R., Weimer, P. J., van Zyl, W. H., and Pretorius, I. S. (2002). "Microbial cellulose utilization: Fundamentals and biotechnology," Microbiol. Mol. Biol. Rev. $66,506-577$. 
Lynd, L.R., Wyman, C.E., and Gerngross, T.U. (1999). "Biocommodity engineering," Biotechnol. Prog. 15, 777-793.

MacFarlane, D. R., Pringle, J. M., Johansson, K. M., Forsyth, S. A., and Forsyth, M. (2006). "Lewis base ionic liquids," Chem. Commun. 18, 1905-1917.

Mazza, M., Catana, D. A., Vaca-Garcia, C., and Cecutti, C. (2009). "Influence of water on the dissolution of cellulose in selected ionic liquids," Cellulose 16, 207-215.

Miller, G.L. (1959). "Use of dinitrosalicylic acid reagent for determination of reducing sugar," Anal. Chem. 31, 426-428.

Moulthrop, J. S., Swatloski, R. P., Moyna, G., and Rogers, R. D. (2005). "Highresolution ${ }^{13} \mathrm{C}$ NMR studies of cellulose and cellulose oligomers in ionic liquid solutions," Chem. Commun. 12, 1557-1559.

Murugesan, S., and Linhardt, R. J. (2005). "Ionic liquids in carbohydrate chemistrycurrent trends and future directions," Curr. Org. Synth. 2, 437-451.

Nelson, M. L., and O'Connor, R. T. (1964). "Relation of certain infrared bands to cellulose crystallinity and crystal lattice type. Part II. A new infrared ratio for estimation of crystallinity in celluloses I and II," J. Appl. Polym. Sci. 8, 1325-1341.

O’Connor, R. T., DuPré, E. F., and Mitcham, D. (1958). “Applications of infrared absorption spectroscopy to investigations of cotton and modified cottons. Part I: physical and crystalline modifications and oxidation," Textile Res. J. 28, 382-392.

Oh, S. Y., Dong, I. Y., Shin, Y., Hwan, C. K., Hak, Y. K., Yong, S. C., Won, H. P., and Ji, H. Y. (2005). "Crystalline structure analysis of cellulose treated with sodium hydroxide and carbon dioxide by means of X-ray diffraction and FTIR spectroscopy," Carbohydr. Res. 340, 2376-2391.

Olivier-Bourbigou, H., Magna, L., and Morvan, D. (2010). "Ionic liquids and catalysis: Recent progress from knowledge to applications," Appl. Catal. A 373, 1-56.

Popa, V. I., Spiridon, I., and Bobu, E. (1996). "Some preliminary data on the use of Asclepias syriaca seed hairs in pulp and paper manufacture," Cell. Chem. Technol. 30, 223-227.

Remsing, R. C., Swatloski, R. P., Rogers, R. D., and Moyna, G. (2006). "Mechanism of cellulose dissolution in the ionic liquid 1-N-butyl-3-methylimidazolium chloride: A ${ }^{13} \mathrm{C}$ and ${ }^{35 / 37} \mathrm{Cl}$ NMR relaxation study on model systems," Chem. Commun. 12, 12711273.

Široký, J., Blackburn, R. S., Bechtold, T., Taylor, J., and White, P. (2010). “Attenuated total reflectance Fourier-transform Infrared spectroscopy analysis of crystallinity changes in lyocell following continuous treatment with sodium hydroxide," Cellulose $17,103-115$.

Spiridon, I. (2007). "Modifications of Asclepias syriaca fibers for paper production," Ind. Crops Prod. 26, 265-269.

Swatloski, R. P., Spear, S. K., Holbrey, J. D., and Rogers, R. D. (2002). "Dissolution of cellulose with ionic liquids," J. Am. Chem. Soc. 124, 4974-4975.

Turner, M. B., Spear, S. K., Huddleston, J. G., Holbrey, J. D., and Rogers, R. D. (2003). "Ionic liquid salt-induced inactivation and unfolding of cellulase from Trichoderma reesei," Green Chem. 5, 443-447.

Van Rantwijk, F., and Sheldon, R. A. (2007). "Biocatalysis in ionic liquids," Chem. Rev. 107, 2757-2785. 
Wu, J., Zhang, J., He, J., Ren, Q., and Guo, M. (2004). "Homogeneous acetylation of cellulose in a new ionic liquid," Biomacromolecules 5, 266-268.

Zhang, Y.-H.P., and Lynd, L.R. (2004). "Toward an aggregated understanding of enzymatic hydrolysis of cellulose: Non-complexed cellulase systems," Biotechnol. Bioeng. 88, 797-824.

Zhang, H., Wu, J., Zhang, J., and He, J. (2005). "1-Allyl-3-methylimidazolium chloride room temperature ionic liquid: a new and powerful non-derivatizing solvent for cellulose," Macromolecules 38, 8272-8277.

Zhao, H., Jones, C. I. L., Baker, G. A., Xia, S., Olubajo, O., and Person, V. N. (2009). "Regenerating cellulose from ionic liquids for an accelerated enzymatic hydrolysis," J. Biotechnol. 139, 47-54.

Zhbankov, R. G., Firsov, S. P., Korolik, E. V., Petrov, P. T., Lapkovski, M. P., Tsarenkov, V. M., Marchewka, M. K., and Ratajczak, H. (2000). "Vibrational spectra and the structure of medical biopolymers," J. Mol. Structure 555, 85-96.

Zhu, S. D.,Wu, Y. X., Chen, Q. M., Yu, Z. N., Wang, C. W., Jin, S. W., Ding, Y. G., and Wu, G. (2006). "Dissolution of cellulose with ionic liquids and its application: A mini-review," Green Chem. 8, 325-327.

Article submitted: August 26, 2010; Peer review completed: October 10, 2010; Revised version received: November 3, 2010; Further corrections: November 18, 2010; Article accepted: December 10, 2010; Published: December 20, 2010; Correction of year shown in footer (from incorrect 2010 to correct 2011): May 5, 2011. 